\title{
See me here, see me there
}

Fifty years ago, a physics student dissatisfied with the standard view of quantum mechanics came up with a radical new interpretation. Mark Buchanan reports on the ensuing debate.

n 1957, a young physicist from Princeton University published his first paper - it went virtually unnoticed - and then disappeared from academia. He worked as an engineer and analyst in the defence industry until he died in 1982, at the age of 51. But Hugh Everett's lasting contribution to science, some physicists argue, stands far out of proportion to his paper tally. His first paper, they say, provided a new way to understand one of the most enduring puzzles of quantum physics.

Quantum theory has had many spectacular successes, but physicists have always been unsettled by its logical consistency. The theory, exemplified in Schrödinger's wave equation, insists that quantum particles such as electrons evolve into weird states of 'superposition' in which, among other possibilities, they can be in two places at once. This equation helped to explain the behaviour of atoms, but ordinary objects such as chairs are also made of quantum particles, so why don't we ever see them in two places at once?

One answer to this conundrum - as expressed in the late 1920s by Niels Bohr and Werner Heisenberg in their famous Copenhagen interpretation - was that we don't see these weird states because they collapse whenever we try to measure them. Everett, in bold contrast, suggested another solution - that the superpositions do affect our world, we simply don't notice them. As he pointed out, the maths of quantum theory suggests that when we encounter an object of superposition of say, here and there, that superposition draws us in too; splitting us into one being who sees the object here, and another who sees it there. In essence, as a later physicist put it, Everett claimed that quantum physics reveals a Universe that perpetually splits into "many worlds" coexisting side by side.

This idea was largely dismissed as being too weird, and many alternatives have been

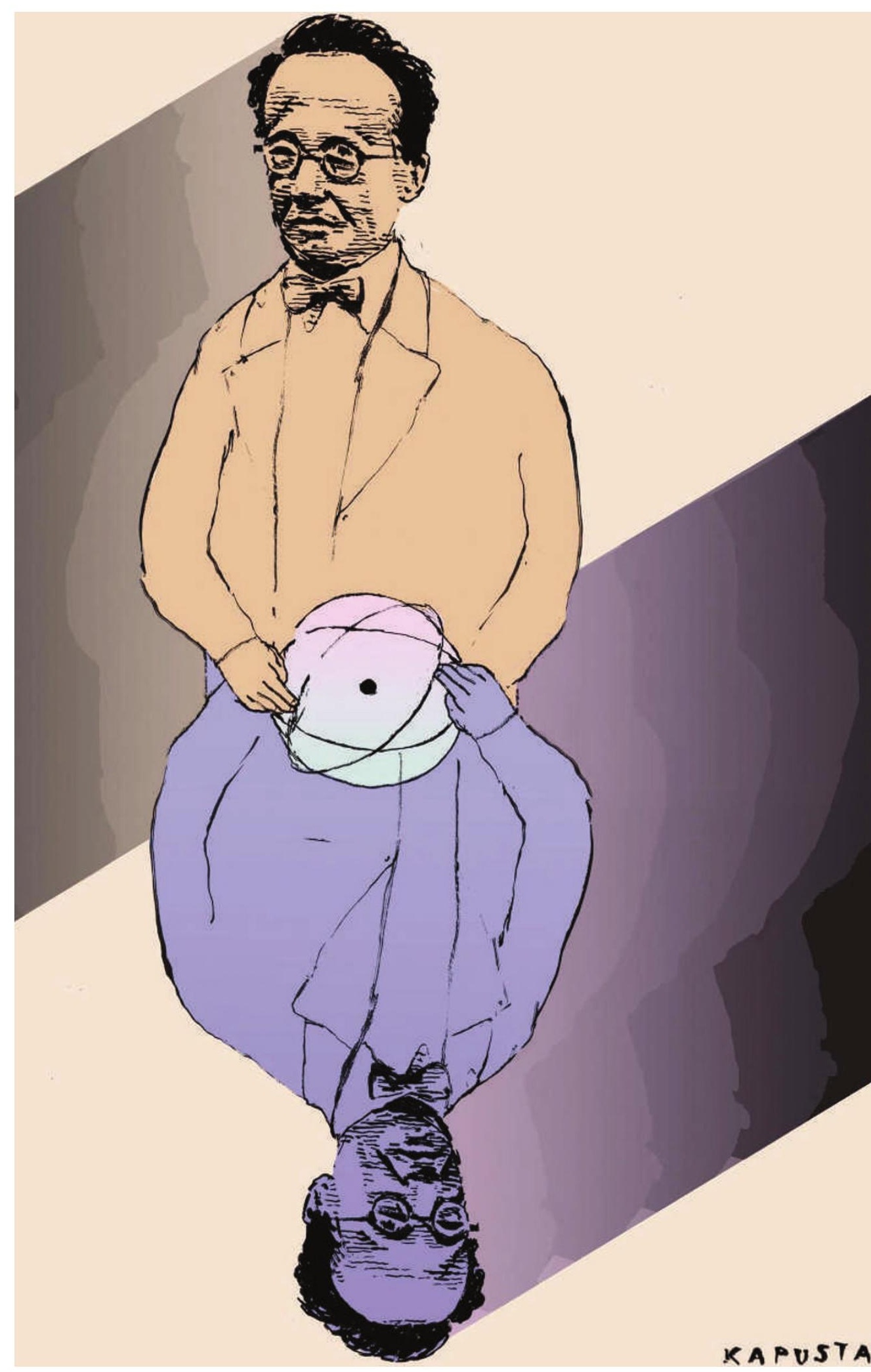


suggested since. Empirical tests are unhelpful. Critics argue that experiments alone cannot distinguish between the many-worlds idea and some of the alternatives. "If experiments continue to verify quantum theory, we're going to be in a very difficult position, having to decide between theories not on evidence but on something else," says physicist and philosopher David Albert from the University of Columbia in New York. Supporters, though, argue that experiments are indeed helping their case - by continuing to find no evidence for the mysterious 'collapse' required by the Copenhagen interpretation.

Later this year, a select gathering of a few dozen physicists, philosophers and mathematicians will meet at two conferences in Canada and England to explore the status of Everett's ideas, 50 years on. "The idea," says philosopher of science Simon Saunders of the University of Oxford, and co-organizer of the meeting in England, "is to assemble the key arguments for and against the interpretation, and to come to a verdict - if not on the interpretation itself, then at least on the precise nature of its strengths and weaknesses."

\section{Out of control}

The interpretations of quantum theory - and the puzzle over why we never experience superpositions - has challenged physicists ever since the theory became indispensible for explaining atomic and nuclear physics early in the twentieth century. Bohr and the early interpreters of quantum theory got around this puzzle simply by dividing the world into classical and quantum parts, and supposing that quantum theory applied only to the latter. By their thinking, electrons and other quantum particles should evolve with quantum wavelike dynamics, but only until they interact with a classical object, such as a measuring device, at which point any superpositions would collapse, leaving just one outcome. Bohr pointed to the inherently uncontrollable nature of quantum interactions to account for the unpredictability of actual measurements.

In the 1930s, Hungarian mathematician John von Neumann formalized these ideas in his 'collapse postulate', which subsequently became one of the standard rules of quantum mechanics. Using the postulate, one can calculate the probabilities of the different outcomes when a 'measurement' takes place, with incredible accuracy. But quantum theory still does not explain in precise detail when or why collapse should occur. For all its success, the theory seemed incomplete. "As they appear in the textbooks", says physicist Wojciech Zurek of the Los Alamos National Laboratory in New Mexico, "the axioms of quantum theory are inconsistent."

\section{Bridging the divide}

And for many physicists, most prominently Albert Einstein, Bohr's split between classical and quantum regimes introduced an unacceptable and arbitrary divide in physicists' view of the world. Quantum theory ought to be able to make sense of classical measuring devices, because these too are made of quantum particles. Restoring such wholeness has been the
Everett's alternative takes yet another perspective. He simply insisted that collapse never happens, and that everything in the Universe always runs in tune with the wavelike dynamics of the Schrödinger equation. We seem to see things that look like collapse, he argued, only because we're part of the quantum world too, and so get caught up in its branching superpositions. Take quantum theory seriously, Everett insisted, and it supplies its own interpretation of reality - with the Universe splitting into multiple parallel worlds.

"In this view," says theorist David Deutsch from the University of Oxford, "our Universe is only a tiny facet of a larger multiverse, a highly structured object that contains many aim of quantum theorists ever since.

Starting in the 1950s, with American physicist David Bohm, theorists have tried their best to develop a coherent quantum theory. Bohm proposed a so-called hidden-variables interpretation, in which quantum particles have unique positions and velocities at all times. Bohm's theory doesn't require collapse, but its unusual mathematical structure - and the apparent impossibility of ever measuring these hidden variables experimentally — has made it attractive to only a few physicists.

Over the past two decades, various researchers have proposed interpretations that effectively side with Bohr's view, but make it more specific. These theories propose that Schrödinger's equation should be modified so that superpositions collapse naturally and, importantly, very rapidly in large, classical objects - thereby providing a more precise explanation of why we never see such superpositions at our level. They do away with the collapse postulate by effectively bringing it into the Schrödinger equation in a mathematically consistent way. universes. Everything in

our Universe - including you and me, every atom and every galaxy - has counterparts in these other universes."

All three approaches have their adherents, but for what seems to be a growing number of physicists, especially those working in quantum information and cosmology, it is Everett's alternative that wins out. "These fields follow the idea that there's nothing wrong with taking quantum theory to its logical limits," says Zurek. "And it was Everett who gave us the permission to do this."

\section{Two-timing particles}

Everett's idea is gaining popularity, at least in part, because quantum theorists have made progress in solving some of its earlier problems, which were not long ago thought to be fatal. One problem is that although Everett's view implies that the world splits into multiple branches, it does not, on its own, give a clear recipe for how this branching should take place. In standard quantum theory, for example, an electron in a fuzzy ill-defined 
state can be thought of as a superposition of multiple states of well-defined position, or, alternatively, as a superposition of states with fuzzy positions but well-defined velocities. Neither picture is more correct than the other. Similarly, Everett's theory doesn't provide an obvious right way to identify the multiple co-existing branches that make up a superposition.

\section{Deceptive behaviour}

A body of ideas known as decoherence theory was developed by physicist Dieter Zeh of the University of Heidelberg in Germany in the 1970s, and has been expanded on by Zurek since then. The theory notes that because quantum systems interact with their environment they do not remain in superpositions for long, but instead tend to 'decohere'. In effect, environmental interactions make quantum systems behave as if their superpositions had collapsed, when in fact they have just become so entangled with the surrounding environment that no experiment, for practical purposes, would be able to detect them; and this is especially true of systems that involve many particles.

Many physicists suggest that, when coupled with decoherence, the many-worlds idea provides an attractive package. That's because decoherence singles out the familiar states of classical objects as being more robust and therefore observable. The idea even has some experimental support, and seems to remove much ambiguity from the many-worlds view.

Another long-standing problem for Everett's view is probability, one of the bedrocks of quantum physics. Using the collapse postulate, the magnitude of the wavefunction can be used to calculate probabilities for the position or velocity of an electron. But the many-worlds perspective, with its insistence on the never-collapsing evolution of quantum systems, says nothing at all about probabilities. "In Everett," says philosopher of physics Wayne Myrvold of the University of Western Ontario in Canada, "there seems to be no room for statements of probability at all."

Even so, recent work by Deutsch and by philosopher of physics David Wallace, also at the University of Oxford, suggests that quantum probabilities can find a way into the theory - through consideration of how human brains, or those of other organisms, register changes in their environments.

They used decision theory - the logical science of optimal decision making - to explore how an individual living in a branching manyworlds universe would behave if he or she were trying to anticipate real-world outcomes as accurately as possible. They conclude that such individuals, confronting new outcomes as they entered new branches, would be driven to assigning probabilities to those branches, and would end up using the very same probabilities prescribed in the collapse postulate.

Although not everyone is convinced, some physicists think that Deutsch and Wallace succeeded in showing how the probabilities of quantum theory arise naturally from Everett's theory. "I'm not fully convinced that it all makes sense," says Myrvold, "but great strides have been made, and it's less implausible than I used to think."

\section{Bigger and better}

Everett enthusiasts also point to the increasing sophistication of modern experiments, which continue to verify the predictions of quantum theory, with no known exceptions. Using the techniques of atom trapping and quantum optics, experimentalists have observed quantum superpositions in systems containing more and more particles, as Everett's theory predicts ought to be possible. "The many-worlds view is slowly becoming the majority preference among physicists,"

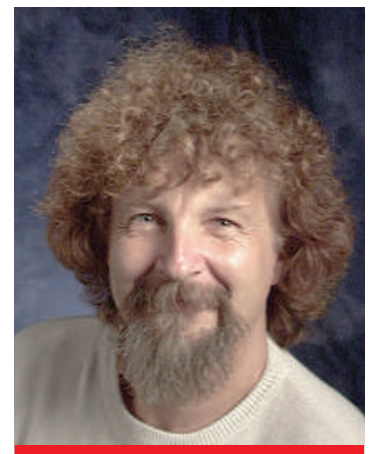

"It was Everett who gave us the permission to take quantum theory to its logical limits." - Wojciech Zurek says Tony Sudbery, a mathematician at the University of York, UK, "at least in part because we see quantum theory working, without any hint of collapse, for systems that are larger and larger."

But Albert says that the idea that experiments lend support to the many-worlds view is wishful thinking. As he points out, all experiments that verify quantum mechanics are consistent with the many-worlds theory and with several of the alternatives, including Bohm's interpretation of hidden variables. Observing wavefunction collapse in an experiment would contradict the Everett view, but the failure to do so might simply reflect the dif- many as $10^{6}$ particles.

ficulty of doing controlled experiments on quantum superpositions.

Physicists hope that future experiments will challenge the idea of wavefunction collapse more directly by testing some of the theories that modify the Schrödinger equation. "Unfortunately," Albert says, "at this point, we're really very far from being able to do the necessary experiments." The current record for a quantum superposition is around 1,000 particles, although some researchers have bold plans to search for superposition collapse in a macroscopic object, perhaps a virus containing as

\section{Seeing is believing}

This is where the debate at the two meetings this year is likely to kick off: with arguments over what experiments can ever prove and whether theorists have really fixed all the conceptual holes in the many-worlds perspective. Although both events have been organized by Everett enthusiasts, they're unlikely to be onesided affairs, as many researchers still have grave doubts about his ideas. "Even if one accepts 'many worlds', says physicist Roger Penrose of the University of Oxford, "one needs a theory of consciousness, in effect, to explain the physics that we actually perceive going on in the world." Without it, he argues, the many-worlds theory is putting the cart before the horse.

Even Albert, who is lukewarm about the many-worlds view but positive about recent theoretical work, says, "My own guess is that, in the end, it's not going to work. But at least the people nowadays visibly understand what the worries and problems are."

Saunders and other Everett supporters suggest that those who hold out against the manyworlds picture are often driven, whether they say so or not, by an instinctive dislike of its non-intuitive consequences. "Quantum theory under the Everett interpretation is fantastic - too fantastic for the great majority of physicists to take seriously," Saunders says. But however they do it, as physicists struggle with the many-worlds interpretation, and the many alternatives, they are at least paying respect to the central idea of Everett's thesis: to take quantum theory very seriously indeed.

Mark Buchanan is a science writer based in Europe. His latest book is The Social Atom.

See Editorial, page 1, and Commentary, page 23. 